\title{
LA RELACIÓN DE OBJETO DEL SELF EN EL TRATAMIENTO PSICOANALÍTICO ${ }^{1}$
}

\author{
Howard A. Bacal, M.D. ${ }^{2}$ \\ Los Ángeles, CA, USA
}

Todos concuerdan, en general, en que el concepto de objeto del self es la piedra angular de la perspectiva de la psicología del self en el psicoanálisis. Es tan central este término que Basch ha sugerido que la adecuada designación para la psicología del self debería ser "la teoría motivacional de objeto del self". En este trabajo, dirijo la atención hacia la naturaleza relacional de la experiencia de objeto del self en el tratamiento psicoanalítico. Identifico la relación de objeto del self como algo más que un simple fondo estático de la experiencia del paciente sobre el cual se conduce la terapia de forma efectiva. Sugiero que es una experiencia dinámica y continua que surge de una compleja interacción entre el analista y el analizando y que es decisiva para que continúe el proceso terapéutico. También sugiero que es experimentado de muchas maneras, muy legítimamente, por ambos participes, lo que tendrá un efecto significativo en el curso de la terapia.

\section{Palabras clave: Psicología del Self, Proceso de tratamiento, Psicoanálisis}

It is generally agreed that the selfobject concept is the cornerstone of the self-psychological perspective in psychoanalysis. So central is it that Basch has suggested that the proper designation of self psychology should be "selfobject theory of motivation". In this paper, I draw attention to the relational nature of the selfobject experience in psychoanalytic treatment. I identify the selfobject relationship as more than simply a static background experience of the patient against which therapy is effectively conducted. I suggest that it is a continuous dynamic experience that arises from a complex interaction between analyst and analysand and is integral to the ongoing therapeutic process. I also suggest that it is variously experienced, quite legitimately, by both participants and that this will have a significant effect on the course of the therapy.

Key Words: Self Psychology, Treatment Process, Psychoanalysis.

English Title: The Selfobject Relationship in Psychoanalytic Treatment

Cita bibliográfica / Reference citation:

Bacal, H.A. (2017). La Relación de Objeto del Self en el Tratamiento Psicoanalítico. Clínica e Investigación Relacional, 11 (1): 11-21. [ISSN 1988-2939] [Recuperado de www.ceir.info ] DOI: 10.21110/19882939.2017.11010101

\footnotetext{
${ }_{1}^{1}$ Publicado originalmente en 1994: The Selfobject Relationship in Psychoanalytic Treatment. Progr. Self Psychol., 10:2130. Traducido, con autorización, del original inglés por N. Monserrat Gómez García, revisada por Alejandro Ávila. 2 HOWARD A. BACAL, M.D., es Psicoanalista y Psicoterapeuta (Los Ángeles, CA, USA). Analista Didacta y Supervisor del Institute of Contemporary Psychoanalysis, Los Ángeles; Analista Didacta y Supervisor del New Center for Psychoanalysis, Los Ángeles; Analista Didacta y Supervisor del Institute for the Psychoanalytic Study of Subjectivity, New York. Ha presidido las conferencias internacionales anuales de la Psicología del Self de 1984, 1993, 2001, 2004, 2007 y es miembro del Consejo Asesor de la IAPSP. Ha publicado muy numerosos y destacados trabajos, entre ellos sus libros: Optimal Responsiveness. How Therapist heal their patients (H. Bacal, Ed., Jason Aronson, 1998) y The Power of Specificity in Psychotherapy. When therapy works and when it doesn't (H. Bacal con L. Carlton, Jason Aronson, 2011).
} 
Kohut formuló el concepto de objeto del self ${ }^{1}$ cuando comenzó a percibir los complejos estados mentales de sus pacientes en sus propias experiencias subjetivas, de manera consistente, a través de lo que él llamaba introspección vicaria, o empatía. Lo que descubrió fue que sus pacientes no parecían sentirlo a él como un objeto hacia el cual pudieran dirigir sus deseos conflictivos y descargar sus impulsos instintivos; en cambio, parecían necesitar respuestas de él que pudieran evocar y afectar a su propio sentido de self. Él era, como si fuera, un "objeto del self" que (los pacientes) necesitaban sentir de diferentes maneras: para confirmar su sentido de vigor y perfección; como una figura que inspirase calma, infalibilidad y omnipotencia con la cual ellos pudieran fusionarse; y como alguien que estaba silenciosamente presente, pero, en esencia, como ellos mismos. Kohut (1971) observó la aparición de estas configuraciones recurrentes e identificables, a las cuales nombró "estructuras cuasi-transferenciales" (p.25) que indicaban, de diversas maneras, tanto el surgimiento de las necesidades de objeto del self de especularizar, idealizar y gemelaridad, o de utilizar respuestas de alter ego, así como la experiencia de satisfacer esas necesidades, pero también la expectativa de que el analista respondería a esas necesidades. Lo que quiero mostrar aquí es el hecho de que el objeto del self es un concepto multifacético que implica tanto la experiencia de una necesidad y la experiencia de la funcionalidad provista por el otro u el "objeto" ${ }^{2}$, así como la experiencia de una relación y que este concepto está presente desde el comienzo de la psicología del self. Mi concepción de lo que es una relación de objeto del self es que se trata de una experiencia intrapsíquica del otro que puede considerarse como proveedor de funciones esenciales de objeto del self. La cohesión del self es reforzada por la expectativa de que este otro responderá óptimamente a las propias necesidades de objeto del self. Hay un sentimiento de pertenencia de este otro que se asocia con el sentido de ser dueño de su responsividad³ óptima, las cuales, ambas, pueden operar hasta cierto punto desde un nivel inconsciente (ver Bacal y Newman, 1990, p. 233, 252). En otras palabras, una relación de objeto del self es aquella en la cual prevalece un sentido relativamente estable de la disponibilidad del objeto como objeto del self. Hay un vínculo central con el analista en el sentido de que el analista esté con el analizando en la forma que él o ella necesiten que el analista esté.

Mientras que la "transferencia" ${ }^{4}$ de objeto del self se refiere comúnmente a un enlace o vínculo, es decir, como una relación de objeto del self (ver Kohut, 1984, pp. 4952), la única alusión sobre su funcionamiento como relación de objeto del self en la situación clínica ha sido el verla como el contexto necesario en el cual se lleva a cabo el trabajo terapéutico que se da en primer plano (ver Stolorow, Brandchaft y Atwood, 1987, p.26). Recientemente, sin embargo, la relación de objeto del self está siendo reconocida 
no meramente como una conexión vaga, amistosa o de apoyo, sino como una experiencia que se encuentra en el centro del proceso terapéutico. Así, Wolf (1988), en su reciente descripción del objeto del self, lo considera como "siendo ni self ni objeto, sino el aspecto subjetivo de una función auto sostenible realizada por una relación del self hacia objetos que por su presencia o actividad evocan y mantienen al self y la experiencia de individualidad" (p. 184, cursivas añadidas). Es también significativo que Stern (1985) considere el objeto del self como un "término (adecuado) para una variedad de relaciones funcionales en curso con otros que son necesarias para proveer de estructuras que regulen y mantengan y/o mejoren la auto-cohesión" (p. 242, cursivas mías). 5

He descrito cómo las ideas de ciertos teóricos británicos de las relaciones objetales anticiparon el aspecto relacional de la experiencia de objeto del self (Bacal, 1987; Bacal y Newman, 1990): El énfasis de lan Suttie sobre la importancia del acompañamiento, la seguridad y la responsividad de la figura materna resuenan fuertemente con el concepto de objeto del self. Algunos aspectos de las relaciones de apego de Bowlby están fuertemente reflejadas en el concepto de objeto del self, tal y como Lichtenberg (1989) había incluido en su esquema de los sistemas motivacionales. El concepto de la figura de apego de Bowlby también reconoce la especificidad del objeto del self. Esto es, que implica la experiencia de la relación con un otro importante: no es que valga cualquier experiencia de objeto del self con quien sea; es aquel terapeuta el que el paciente echa de menos o cuya ausencia desencadena el desmoronamiento del paciente. Balint (1968) y Winnicott (1954), en particular, describen ciertas características de la relacionalidad arcaica que parecen casi idénticas a las de Kohut sobre la fusión arcaica o la relación de objeto del self especular ${ }^{6}$ en pacientes que atraviesan estados regresivos profundos en el análisis. Sin embargo, como tenían a Winnicott, no ha sido hasta hace muy poco que los analistas británicos hayan formalmente reconocido que la psicología del self tuviera algo que ofrecerles. ${ }^{7} \mathrm{Y}$ los psicólogos del self, no solo yo, estamos ahora prestando más atención a algunas de sus ideas: una de las (propuestas) más sofisticadas de la psicología del self es análoga a la concepción de Winnicott sobre que el infante que no puede ser considerado por sí mismo, sino como una parte de la unidad progenitor-infante como se refleja en la premisa de Stolorow y Atwood (1992) sobre el "mito de la mente aislada".

Y todavía, a pesar de los aspectos comunes que hay entre la percepción de ciertos teóricos británicos de las relaciones objetales y de los de la psicología del self, la naturaleza intrínseca de la relación de objeto del self los separa significativamente de cualquier otro concepto relacional en psicoanálisis. Para poder apreciar esto 
completamente, debemos volver a las primeras conceptualizaciones de Kohut en 1971 sobre el objeto del self.

Mientras Kohut (1971) inicialmente pensaba al objeto del self como facilitando la experiencia narcisista, él definió el objeto del self como objetos que "o están siendo usados al servicio del self o son experimentados por sí mismos como parte del self" ( $p$. xiv). También trajo una actitud completamente nueva sobre los conceptos de las necesidades narcisistas y las experiencias narcisistas. Porque las consideraba, no como reflejando una fijación, una defensa o una intensificación de amor a uno mismo, sino como un intento por reparar un déficit del desarrollo a través de una relación con él, pudiendo responder a ellos como psicológicamente legítimos. Aunque Freud había conferido un sentido de legitimidad al análisis del narcisismo (ver Wolf, 1988, pp. 6-8), abriendo así un importante y nuevo territorio para el trabajo terapéutico, hasta entonces, los analistas, incluyendo los teóricos británicos de las relaciones objetales, solo aceptaban las necesidades arcaicas del paciente cuando estos se encontrasen en estados regresivos graves; y respondían a anhelos arcaicos de los demás pacientes de forma que enfatizaban lo inapropiado de su edad de desarrollo, mostrando lo que Kohut vino a llamar "madurez-moralidad". Kohut, en contraste, introdujo el concepto de objeto del self para denotar las necesidades de objeto del self del analizando, sus experiencias y expectativas de la respuesta del otro como psicológicamente apropiadas, o legítimas, sin hacer referencia al nivel o grado de regresión del paciente. ${ }^{8} \mathrm{Al}$ hacer eso, transformaba la manera en la que vemos la relación psicoanalítica y la forma en que tratamos a nuestros pacientes.

No obstante, Kohut fue mucho más allá cuando también caracterizó la experiencia de objeto del self como la expectativa del analizando de controlar al otro y la función que cumple, siendo más (parecido) como el control que los adultos esperarían tener sobre su propio cuerpo y mente (ver Kohut, 1971, p. 32). Subrayo la palabra expectativa porque creo que sus implicaciones han sido poco apreciadas. Kohut, en efecto, validó el sentido del paciente de sentirse naturalmente con todo el derecho de disponer de ciertas respuestas humanas básicas del analista como una figura parental en la transferencia. Hoy estamos menos interesados en la pregunta de si tal vez los analizandos sienten al objeto del self, o no, como parte de su self físico y, desde el trabajo de Stern, tendemos a pensar que en realidad no lo sienten así. Concuerdo con la idea de Morton Shane (1991) de que la inclusión psicológica del análisis, en el sentido que tiene el paciente de su self, es tan intrínseca para el concepto de objeto del self que se usa ahora, como lo era para la conceptualización de Kohut en 1971, pero esto lo reconsideraría en el sentido de que los pacientes sienten que tienen un sentido básico de 
derecho sobre la respuesta de objeto del self del analista. Este sentimiento de derecho natural sobre la respuesta de otro significativo se debe distinguir de las expresiones de derecho urgentes, intensas, agitadas, controladoras y algunas veces extravagantes que, de hecho, esconden una deficiencia o alteración en el sentido natural de derecho sobre algo. ${ }^{9}$ Creo que su restauración y su incrementada fuerza y estabilidad son los mayores determinantes de la disminución en la intensidad, urgencia y ansiedad asociada con la experiencia de las necesidades del objeto del self a través del tiempo; en otras palabras, su desarrollo se relaciona con la maduración de las relaciones de objeto del self. Si uno tiene una expectativa confiable de que se tiene derecho sobre las relaciones de objeto del self ${ }^{10}$ con los otros significativos, el self se fortalece y se siente más preparado para responder recíprocamente a las comparables necesidades de objeto del self de los otros.

En pacientes con un self perturbado, este sentido básico de derecho está seriamente afectado y puede aportar significativamente a sentimientos duraderos de ser irremediablemente malo e inadecuado. Sin este sentido de derecho, el paciente se siente psicológicamente solo, una experiencia que algunas veces padecen terriblemente las personas con un trastorno de la personalidad narcisista o límite grave. En otras palabras, sugiero que la experiencia de este sentido básico de derecho es una precondición para poder experimentar o sentir una relación de objeto del self, una relación en la cual los pacientes sienten que pueden contar con la respuesta de objeto del self de su analista. Hay para el paciente un importante sentido de ser dueños del otro o que el otro les pertenece y un conexo sentido dentro del self de ser especial; el objeto del self es el objeto del self. Creo que este sentido de pertenencia es también el núcleo de lo que ha sido llamado "confianza básica".

En otra parte describí el efecto terapéutico del análisis psicológico del self como una "experiencia correctiva del objeto del self" (Bacal, 1990). El proceso terapéutico por el cual se da esta experiencia es a través del establecimiento y la regulación de la relación de objeto del self, ${ }^{11}$ la cual es, en efecto, una creación conjunta por parte del analista y del analizando. Equiparando el proceso terapéutico con la creación mutuamente evolutiva de la relación de objeto del self replantea una perspectiva que otros han desarrollado de diferentes maneras. Como Lachmann y Beebe (1992) han señalado, varios autores tales como Gill, Hoffman, Mitchell, Stolorow, Brandchaft y Atwood han discutido las contribuciones interactivas del analista y del analizando que dan forma a la transferencia y ellos mismos han propuesto la visión de que la regulación mutua entre las contribuciones de ambos participes puede transformar la experiencia del paciente y su estructura psíquica (ver también, Beebe, Jaffe y Lachmann, 1992). Puntos de vista análogos incluyen el concepto de "diálogo de construcción" de Terman (1988), la 
definición de Paul Tolpin (1988) de la tarea terapéutica como "compromiso afectivo óptimo" y la reciente hipótesis de Lessem y Orange (1992) de que el vínculo emocional que se desarrolla como resultado de las interacciones entre el paciente y el terapeuta constituyen el agente curativo principal del psicoanálisis.

Ahora me gustaría esbozar mi concepción de las contribuciones respectivas del paciente y del analista en la creación de la experiencia de una relación de objeto del self. Al hacerlo, también describo lo que considero como elementos centrales del proceso terapéutico. La relación de objeto del self es, en efecto, experimentada tanto por el analista como por el analizando. Consideremos primero las contribuciones del analizando a su experiencia de esta relación:

1. La transferencia de necesidades insatisfechas motiva a los analizandos, a pesar de sus temores y defensas, a poner al terapeuta en el papel de proveedor de funciones psicológicas esenciales para el sustento del self.

2. También se transfieren hacia el terapeuta antecedentes de relaciones de objeto del self, especialmente de la niñez, que también, incitan al paciente a creer que es posible la relación de objeto del self con el terapeuta. Incluiría aquí el sentido de un derecho a los suministros de objeto del self desde una figura parental, lo que he llamado el sentido natural de sentirse con derecho, lo cual es transferido desde las experiencias de las interacciones tempranas que las confirman.

3. La capacidad de los analizandos para crear en la fantasía una figura que esté contagiada con cualidades que pudieran justificar su confianza también contribuye a su experiencia de una relación de objeto del self con su terapeuta. El ejercicio de esta capacidad es el principal determinante de la experiencia de idealización. En la medida en que la capacidad deriva de una experiencia previa de objeto del self, que podría operar casi como el compañero evocado descrita por Stern (1985), es decir, como un "otro regulador del self [que] se vuelve 'presente' en forma de una memoria activa" (p. 116). Es importante reconocer, sin embargo, que también refleja la imaginación creativa del analizando. He encontrado útil el conceptualizar un "objeto del self fantaseado" cuando la experiencia del paciente de su contribución a la experiencia en la relación con el analista al aparecer el objeto del self está basada enormemente en este proceso, esto es, en la movilización de imagos de objetos del self responsivos que el paciente ya ha creado sustantivamente en la niñez. Estas imagos sirven como substitutos y compensan de experiencias intolerables de privación que los pacientes 
sienten que deben continuar repudiando; sin ellas probablemente la experiencia de una relación de objeto del self no sería posible. Una paciente mía me explicó recientemente, durante un periodo en el que ella se encontraba en condiciones de sentir su desesperación y desesperanza, "Haces una fantasía porque no tienes la cosa real". Esta paciente, y otros, también me enseñaron que cualquier relación de objeto del self que se desarrolla principalmente en base a la fantasía van a ser frágiles $y$, por lo tanto, particularmente susceptibles de ruptura.

La esencia de las contribuciones del analista a la experiencia del paciente de una relación de objeto del self es su responsividad óptima a las (experiencias) del paciente (ver Bacal, 1985, 1990; Bacal y Newmann, 1990). Como sabemos, la responsividad óptima del terapeuta puede tomar diversas formas. Estas incluyen la provisión y/o interpretación de una variedad de funciones verbales y no verbales del objeto del self. También incluyen un deseo por colaborar en la tarea de trabajar a través de rupturas en la relación de objeto del self entre los dos participantes. Podría implicar una actitud indagadora o una silenciosa presencia no indagadora, una confirmación repetitiva, o un reto confrontativo. Su forma va a estar determinada no solo por los temas que el paciente y el analista están trabajado, sino también por la fortaleza del self del paciente y por sus niveles operativos de desarrollo logrados, que Basch $(1991,1992)$ ha descrito.

La habilidad del terapeuta para responder óptimamente al paciente va a estar determinada parcialmente por sus experiencias previas de objetos del self. También esta significativamente influida por su capacidad para una imaginación creativa o fantasía. La moderada idealización del paciente por parte del terapeuta, la cual Kohut creía que era un ingrediente esencial para un tratamiento efectivo, esta probablemente colmada por el ejercicio de esta capacidad. Es como si el terapeuta dijera, "Tú, mi paciente, eres una persona especial que tiene el derecho para estar en la relación especial que tenemos juntos".

La complejidad de las interacciones entre el paciente y el terapeuta que constituyen la experiencia de una relación de objeto del self para el paciente también produce la experiencia del terapeuta de una relación de objeto del self con el paciente (Wolf, 1980). El terapeuta, también, trae necesidades del objeto del self insatisfechas, y experiencias previas del objeto de self, a la relación con su paciente y estas engendran expectativas que el analizando responderá de ciertas maneras. Partiendo del acuerdo de que los participantes se han reunido para (lograr) el beneficio psicológico del paciente, nuestra acostumbrada actitud hacia 
las necesidades de objeto del self del terapeuta es que estas deben de considerarse como contratransferencias que deben ser comprendidas. En la medida en que el terapeuta es fortalecido por sus relaciones extra-analíticas de objeto del self y pueda descentrarse de estas necesidades de objeto del self, él o ella será capaz de contribuir efectivamente (ej. Ser óptimamente responsivo) a la necesidad del paciente para una relación de objeto del self en la terapia. Cuando el analista no puede cumplir (por si mismo) con esta función, recomendamos supervisión o más análisis personal. Esta perspectiva es válida, ética y segura, pero ignora lo que todos sabemos pero que hemos encontrado de alguna manera una discusión incómoda entre nosotros: La experiencia del terapeuta de una relación de objeto de self con el paciente no solo es fuertemente operativa en todas las relaciones terapéuticas, sino también constituye una precondición para que el terapeuta responda de maneras que permitirán al paciente experimentar con él o ella una relación de objeto del self. Los analistas regularmente esperan que los analizandos respondan de diversas maneras que son, de hecho, autosostenedoras o beneficiosas para el terapeuta, así como, p.e. cumplir el compromiso de asistir regularmente a las sucesivas sesiones, por las cuales se pagan honorarios, y sostener un proceso en donde ambos, paciente y terapeuta, esperan que el paciente haga ciertos progresos con el tiempo. Estas son algunas de las evidentes funciones del objeto del self que el analista espera conscientemente del analizando, que por lo general experimentan en sus relaciones de objeto del self con el analizando, y la cual ellos tácitamente, y en algunas ocasiones explícitamente, consideran como precondiciones para que se de un funcionamiento analítico efectivo. Hay otras que operan desde un nivel inconsciente; es probable que gran parte de lo que nosotros llamamos contratransferencia corresponda a la experiencia del analista de frustración en sus necesidades de objeto del self que son ordinariamente satisfechas en la relación de objeto del self con el analizando. Tal vez Stolorow y yo podríamos estar de acuerdo en llamar a esto "respuesta intersubjetiva óptima". En cualquier circunstancia, hay cierta mutualidad esencial o reciprocidad en la relación analítica de objeto del self. Este es un tema que merece más explicación e ilustración, la cual ofreceré en otra ocasión (ver Bacal y Thomson, 1993)12.

\section{REFERENCIAS}

Bacal, H. A. (1985). Optimal responsiveness and the therapeutic process. In: Progr. Self Psychol., Vol. 1, ed. A. Goldberg. New York: Guilford, pp. 202-227. 
Bacal, H. A. (1987). British object-relations theorists and self psychology: Some critical reflections. Int. J. Psycho-Anal., 68:81-98.

Bacal, H. A. (1990). The elements of a corrective selfobject experience. Psychoanal. Inq., 10:347372.

Bacal, H. A. (1991). Notes on the relationship between object relations theory and self psychology. In: The Evolution of Self Psychology: Progr. Self Psychol., Vol. 7, ed. A. Goldberg. Hillsdale, NJ: The Analytic Press, pp. 36-44.

Bacal, H. A. \& Newman, K. (1990). Theories of Object-Relations: Bridges to Self Psychology. New York: Columbia University Press.

Bacal, H. A. \& Thomson, P. (1993). The psychoanalyst's selfobject needs and the effect of their frustration on the treatment: A new view of countertransference. Presented at the 16th Annual Conference on the Psychology of the Self, Toronto, October 31, 1993.

Balint, M. (1968). The Basic Fault. London: Tavistock.

Basch, M. F. (1991). Are selfobjects the only objects? Implications for psychoanalytic technique. In: The Evolution of Self Psychology, Progr. Self Psychol., Vol. 7, ed. A. Goldberg. Hillsdale, NJ: The Analytic Press, pp. 3-15.

Basch, M. F. (1992). The selfobject concept: Clinical implications. Presented at the 15th Annual Conference on the Psychology of the Self, Los Angeles, October 10.

Beebe, B., Jaffe, J. \& Lachmann, F. (1992). A dyadic systems view of communication. In: Relational Psychoanalysis, ed. N. Skolnick \& S. Warshaw. Hillsdale, NJ: The Analytic Press, pp. 61-81.

Kohut, H. (1971). The Analysis of the Self. New York: International Universities Press.

Kohut, H. (1977). The Restoration of the Self. New York: International Universities Press.

Kohut, H. (1984). How Does Analysis Cure? ed. A. Goldberg \& P. Stepansky. Chicago: University of Chicago Press.

Kriegman, G. (1988). Entitlement attitudes: Psychological and therapeutic implications. In: Attitudes of Entitlement, ed. V. Volkan \& T. Rodgers. Charlottesville: University Press of Virginia, pp. 1-21.

Lachmann, F. \& Beebe, B. (1992). Reformulations of early development and transference: Implications for psychic structure formation. In: Psychology and Psychoanalysis, ed. J. Barron, M. Eagle \& D. Wolitzky. Washington, DC: The American Psychoanalytic Association, pp. 133-153.

Lessem, P. \& Orange, D. (1993). Emotional bonds: The therapeutic action of psychoanalysis revisited. Presented at the 16th Annual Conference on the Psychology of the Self, Toronto, October 31.

Levine, H. (1979). The sustaining object relationship. Annu. Psychoanal., 7:203-231. New York: International Universities Press.

Lichtenberg, J. D. (1989). Psychoanalysis and Motivation. Hillsdale, NJ: The Analytic Press.

Mollon, P. (in press). The Fragile Self and the Search for Mirroring: the Structure of Narcissistic Disturbance. London: Whurr. 
Shane, M. (1991). Selfobject or self-regulating other. In: The Evolution of Self Psychology: Progr. Self Psychol., Vol. 7, ed. A. Goldberg. Hillsdale, NJ: The Analytic Press, pp. 31-36.

Stern, D. (1985). The Interpersonal World of the Infant. New York: Basic Books.

Stolorow, R. D. \& Atwood, G. E. (1992). Contexts of Being: The Intersubjective Foundations of Psychological Life. Hillsdale, NJ: The Analytic Press.

Storlow, R. D., Brandchaft, B. \& Atwood, G. E. (1987). Psychoanalytic Treatment: An Intersubjective Approach. Hillsdale, NJ: The Analytic Press.

Terman, D. (1988). Optimum frustration: Structuralization and the therapeutic process. In: Learning From Kohut: Progr. Self Psychol., Vol. 4, ed. A. Goldberg. Hillsdale, NJ: The Analytic Press, pp. 113-125.

Tolpin, P. (1988). Optimal affective engagement: The analyst's role in therapy. In: Learning From Kohut: Progr. Self Psychol., Vol. 4, ed. A. Goldberg. Hillsdale, NJ: The Analytic Press, pp. 160168.

Winnicott, D. W. (1954). Metapsychological and clinical aspects of regression within the psychoanalytical set-up. In: D. W. Winnicott: Collected Papers. London: Tavistock, pp. 278-299.

Wolf, E. (1980). Empathy and countertransference. In: The Future of Psychoanalysis, ed. A. Goldberg. New York: International Universities Press, pp. 309-326.

Wolf, E. (1988). Treating the Self: Elements of Clinical Self Psychology. New York: Guilford.

Original recibido con fecha: $\quad$ 5-1-2017 $\quad$ Revisado: $\quad$ 15-2-2017 $\quad$ Aceptado: 28-2-2017

NOTAS:

\begin{abstract}
${ }^{1}$ Inicialmente, la palabra llevaba guion. Kohut quitó el guion entre self y objeto para "expresar más claramente el hecho de que no estamos lidiando con un constructo ad hoc, sino con un concepto viable el cual esperamos que encuentre un lugar duradero en el pensamiento analítico" (en una carta para el International Journal of Psycho-Analyisis el 9 de septiembre de 1978).

${ }^{2}$ El concepto de objeto del self se utiliza para referirse tanto a ciertas necesidades del self como para las experiencias que están siendo cumplidas por otros. Estas necesidades y experiencias sobre el funcionamiento de los otros incluyen fundamentalmente la evocación del sentido del self y la reconstitución del sentido del self después de que ha sido alterado o perdido. Otros reconocen de manera omnipresente las necesidades y experiencias de objeto del self que incluyen la regulación de la autoestima, la sintonización del afecto, la contención del afecto, la regulación de la tensión, el tranquilizar y el vitalizar. Curiosamente, a estas necesidades y funciones de objeto del self no se les ha dado el estatus de las otras tres. Stolorow (1992) describió recientemente una "función auto-delineada de objeto del self" (pp.27, 49), que probablemente se acepte como igualmente importante que las tres descritas por Kohut.

${ }^{3}$ N.de.T. Véase este concepto en el trabajo que sigue en este dossier (1985: Responsividad óptima...)

4 Puse "transferencia" entre comillas porque creo que la designación inicial de Kohut sobre las transferencias de objeto del self como "estructuras cuasi-transferenciales" (Kohut, 1971, p. 25) es correcta en la medida en que incluyen mucho más que transferencia.

${ }^{5}$ En efecto, el punto de vista de Stern (1985) acerca del infante normal es que "la vida es tan social que la mayoría de las cosas que el infante hace, siente y percibe ocurren en diferentes tipos de relaciones" (p. 118).
\end{abstract}

@ ( Derechos reservados/Copyright de Clínica e investigación Relacional y los autores. Prohibida la reproducción total o parcial sin autorización expresa. Este material es para uso científico y profesional exclusivamente y puede contener información clínica sensible. Los editores no se responsabilizan de los contenidos de los autores. Dirigir las consultas sobre derechos y autorizaciones a ceir@psicoterapiarelacional.es 


\footnotetext{
${ }^{6}$ Para más ilustraciones de análogas conceptualizaciones sobre la relación de objeto del self en los escritos de los teóricos británicos de las relaciones objetales, ver a Bacal y Newman (1990).

7 En el verano de 1992 se llevó a cabo un coloquio en Londres entre los miembros del Grupo Independiente de la Sociedad Psicoanalítica Británica y los miembros del Consejo Nacional para la Psicología del Self (ver también Mollon, en prensa).

${ }^{8}$ Algunos psicólogos del self, como Basch (1991b), conservarían la designación de objeto del self para referirse a las respuestas óptimas que solo el amenazado self requiere del terapeuta y lo distinguirían finamente de la cohesiva relación del self con los objetos. He discutido, por otra parte, que en la práctica siempre estamos lidiando con un self relativamente cohesivo (Bacal, 1991). Este se presenta usualmente como un self relativamente debilitado o fragmentado; sin embargo, nosotros regularmente nos encontramos en situaciones en donde los pacientes que están progresando en análisis y cuyo self se está volviendo más fuerte, paradójicamente necesitan, porque su sentido de self está bajo una incrementada tensión por el momento, que la experiencia responsiva del objeto del self que esté por encima y más allá del nivel habitual para elevarse a nuevas cotas de eficacia y libertad.

9 George Kriegman (1988) ha clasificado actitudes de derecho de forma muy similar a la que estoy describiendo aquí.

${ }^{10}$ Comparar con la "relación objetal sostenida" de Howard Levine (1979).

${ }^{11}$ En cierto sentido, Kohut sostuvo una visión comparable. Él creía que no era la interpretación la que curaba al paciente, sino que es la relación terapéutica la que llevaba al vínculo empático entre el paciente y el analista. Él entendió el proceso como portador de micro relaciones entre el self y sus objetos del self que llevan a la construcción de una estructura psicológica a través del proceso de transmutar internacionalizaciones de frustraciones optimas (ver Kohut, 1977, pp. 31-32). Concuerdo con Kohut en que una terapia efectiva conlleva la experiencia de la relación del self con sus objetos del self, pero veo algo diferente la génesis de esta relación.

${ }^{12}$ N.d.T. Este trabajo ha sido traducido al castellano y publicado en la revista Intercambios.
} 